\title{
The Adoption of an Online Journal Club to Improve Research Dissemination and Social Media Engagement Among Hospitalists
}

\author{
Charlie M. Wray, DO, MS $1,2, *$, Andrew D. Auerbach, MD, MPH ${ }^{2,3}$, Vineet M. Arora, MD, MAPP4
}

'Division of Hospital Medicine, San Francisco Veterans Affairs Medical Center, San Francisco, California; ${ }^{2}$ University of California, San Francisco, Department of Medicine, San Francisco, California; ${ }^{3}$ Division of Hospital Medicine, University of California, San Francisco, California; ${ }^{4}$ Section of General Internal Medicine, University of Chicago, Chicago, Illinois.

BACKGROUND: Twitter-based journal clubs are intended to connect clinicians, educators, and researchers to discuss recent research and aid in dissemination of results. The Journal of Hospital Medicine (JHM) began producing a Twitter-based journal club, \#JHMChat, in 2015.

OBJECTIVE: To describe the implementation and assess the impact of a journal-sponsored, Twitter-based journal club on Twitter and journal metrics.

INTERVENTION: Each \#JHMChat focused on a recently published JHM article, was moderated by a social media editor, and included one study author or guest.

MEASUREMENTS: The total number of participants, tweets, tweets/participant, impressions, page views, and change in the Altmetric score were assessed after each session. Thematic analysis of each article was conducted, and post-chat surveys of participating authors and participant responses to continuing medical education surveys were reviewed.
RESULTS: Seventeen Twitter-based chats were held: seven (47\%) focused on value, six (40\%) targeted clinical issues, and four (27\%) focused on education. On average, we found 2.17 ( $\pm 0.583 \mathrm{SD}$ ) million impressions/session, 499 ( $\pm 129 \mathrm{SD}$ ) total tweets/session, and 73 ( $\pm 24 \mathrm{SD}$ ) participants/session. Value-based care articles had the greatest number of impressions ( $2.61 \pm 0.55$ million) and participants $(90 \pm 12)$. The mean increase in the Altmetric score was 14 points $( \pm 12)$, with medical education-themed articles garnering the greatest change (mean increase of 32). Page views were noted to have increased similarly to levels of electronic Table of Content releases. Authors and participants believed \#JHMChat was a valuable experience and rated it highly on post-chat evaluations.

CONCLUSION: Online journal clubs appear to increase awareness and uptake of journal article results and are considered a useful tool by participants. Journal of Hospital Medicine 2018;13:764-769. Published online first July 25, 2018. (C) 2018 Society of Hospital Medicine linicians, educators, and medical journals are increasingly using the social media outlet, Twitter, as a medium to connect and engage with their colleagues. In particular, online journal clubs have created a space for the timely discussion of research, creation of online communities, and dissemination of research.

Social media-based journal clubs are thought to be one way in which journals can leverage the power of social networks so that researchers can engage with a diverse range of end users $^{4}$ (including bedside clinicians, administrators, and patients). Several examples of these models exist. For example, \#GeriMedJC acts as a complimentary, synchronous chat that takes

\footnotetext{
*Address for correspondence: Charlie M. Wray, DO, MS, San Francisco Veterans Affairs Medical Center, University of California, San Francisco, 4150 Clement Street, San Francisco, CA 94121; Telephone: 415-595-9662; Fax: 415-221-4810; E-mail: Charlie.Wray@ucsf.edu.

Additional Supporting Information may be found in the online version of this article.
}

Received: December 18, 2017; Revised: March 12, 2018 ;

Accepted: March 15, 2018

(c) 2018 Society of Hospital Medicine DOI 10.12788/jhm.2987 place at the same time as a live, in-person journal club. \#NephJC offers multiple one-hour chats per month and provides an in-depth summary and analysis of each article, while \#UroJC is an asynchronous discussion that takes place over 48 hours. Few data exist to describe whether any of these programs produce measurable improvements in indicators of engagement or dissemination of results.

In 2015, the Journal of Hospital Medicine (JHM) began producing a Twitter-based journal club as a means to connect and engage the Hospital Medicine community and allow for discussion and rapid exchange of information and opinions around a specific clinical topic. This study aims to describe the implementation of the first Journal-sponsored, Twitter-based online journal club and ascertain its impact on both Twitter and journal metrics.

\section{METHODS}

\#JHMChat was launched in October 2015, and was initially held every two to three months until January 2017, when chats began to take place monthly. Each one-hour chat focused on a recently published article in JHM, was moderated by a JHM social media editor (C.M.W., V.M.A.), and included at least one study author or guest expert. Articles were chosen by the so- 
TABLE 1. Engagement and Dissemination Metrics.

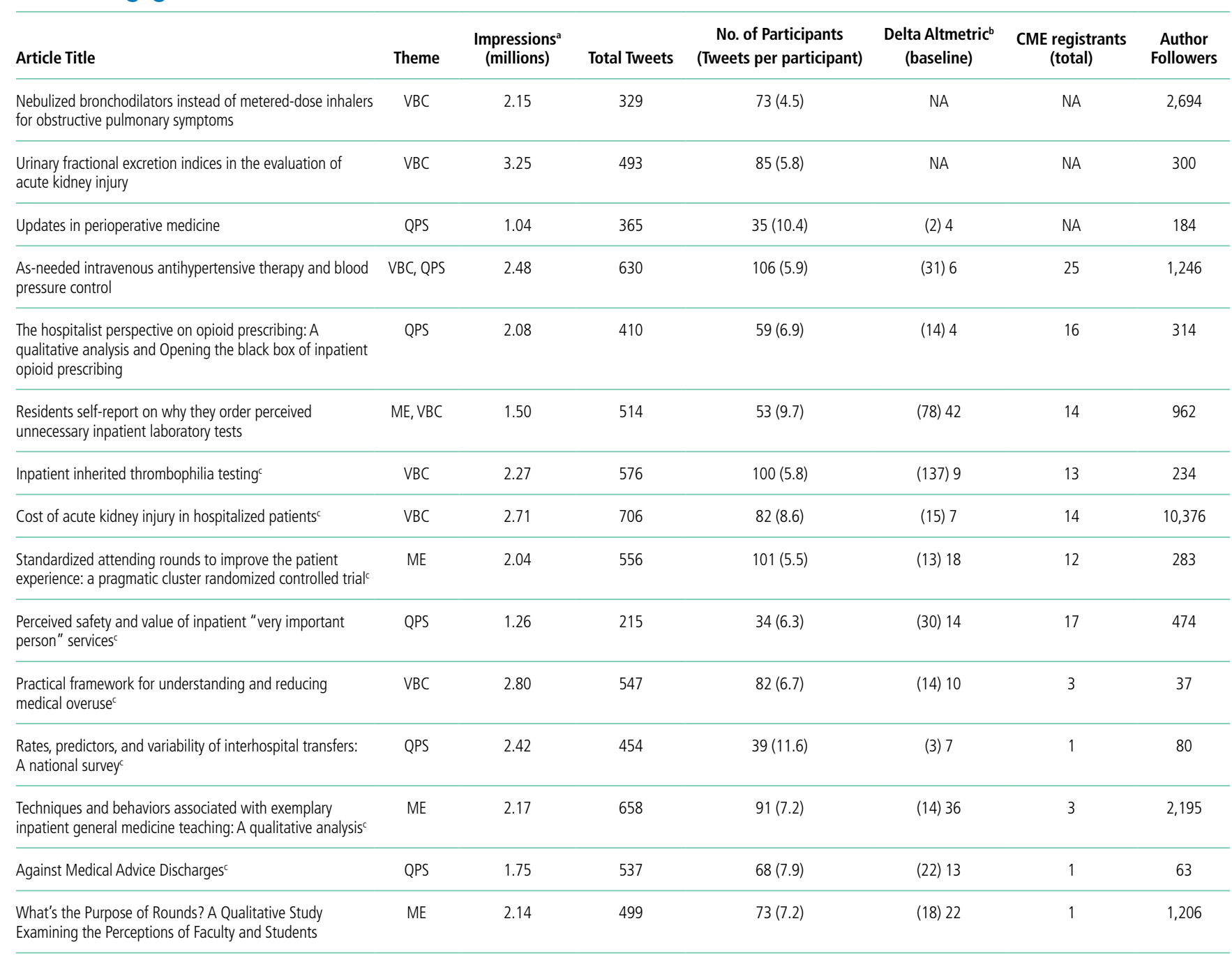

Topics are listed chronologically (top to bottom).

almpressions: calculated by the number of tweets from each participant multiplied with number of followers that participant currently had then summed up for all participants

${ }^{b}$ Delta Altmetric was measured as the difference in Altmetrics score between the day of the discussion and two weeks later. Delta Altmetric was calculated as the difference between the score on the day of the session and two weeks following the respective session, with higher scores indicating greater global online discussion

Indicates the use of a Visual Abstract for promotion on social media.

Abbreviations: CME, Continual Medical Education; ME: Medical Education; QPS, Quality and Patient Safety; VBC, Value-based Care.

cial media editors based on the following criteria: (1) attractiveness to possible participants, (2) providing topic variety within the journal club series, and (3) sustainability and topic conduciveness to the online chat model. Chats were held at 9 PM EST in order to engage hospitalists across all US time zones and on different days to accommodate authors' availability. All sessions were framed by three to four questions intended to encourage discussion and presented to chat participants at spaced intervals so as to stimulate a current of responses.

Chats were promoted by way of the JHM (@JHospMedicine, 3,400 followers) and Society of Hospital Medicine (SHM; @SHMLive, 5800 followers) Twitter feeds beginning one month prior to each session. Visual Abstracts ${ }^{5,6}$ were used to publicize the sessions, also via Twitter, starting in February 2017.

Continuing Medical Education (CME) credits were offered through the SHM to registered participants, starting in July 2016. ${ }^{7}$ All sessions were cosponsored by the American Board of Internal Medicine (ABIM) Foundation and the Costs of Care Organization, a nonprofit organization aimed at improving healthcare value.

\section{Twitter Metrics}

After each session, the following Twitter-based engagement metrics were obtained using the Symplur ${ }^{\circledast}$ Healthcare Hashtag project $^{8}{ }^{\text {total }}$ number of participants and tweets, tweets/participant, and total impressions (calculated as the number of tweets from each participant multiplied by the number of followers that participant currently had then summed up for all participants). Simply put, impressions can also be thought of as the number of times a single Tweet makes it into someone else's Twitter feed. 


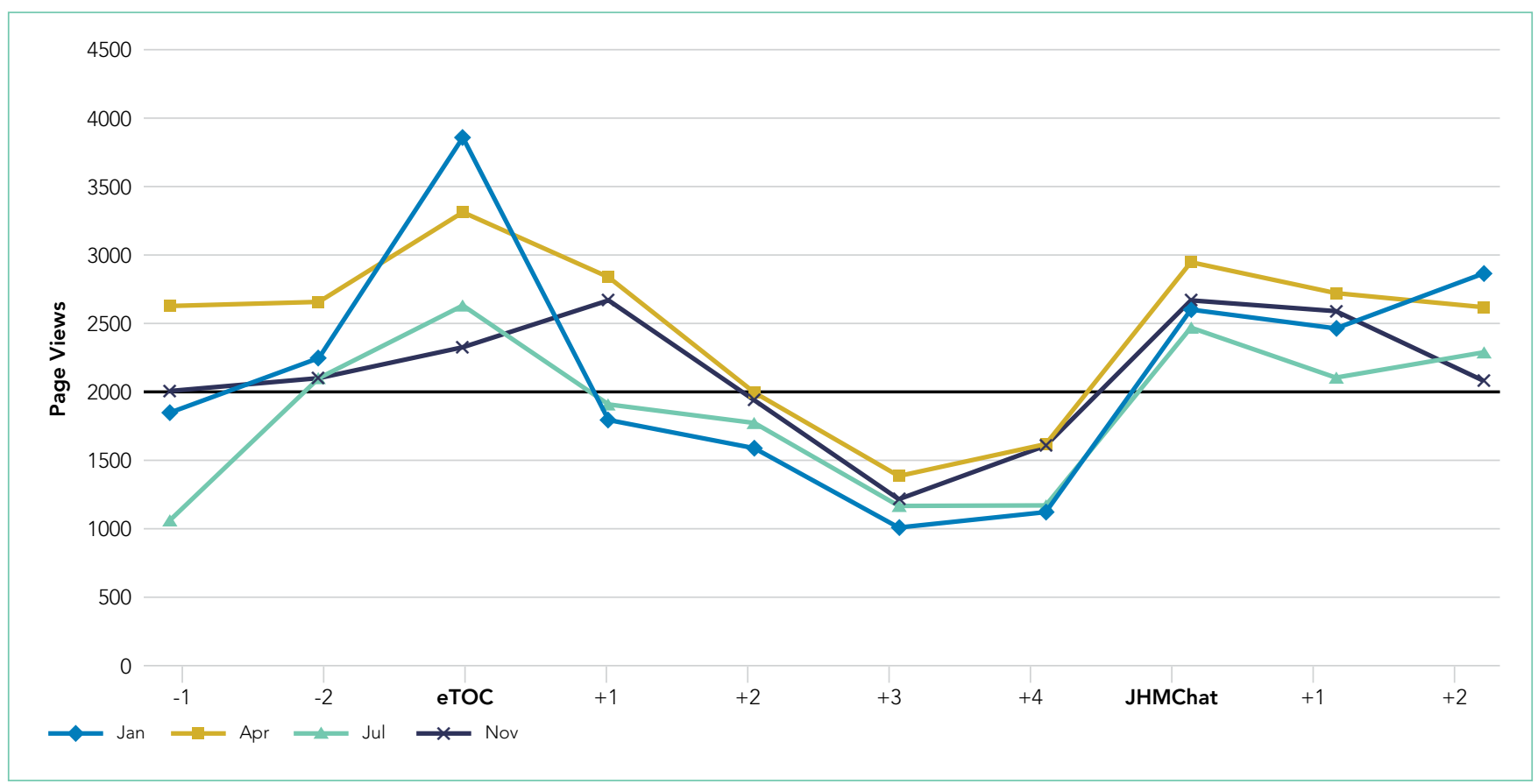

FIG. Journal of Hospital Medicine page views following \#JHMChat and electronic Table of Contents (eTOC) release. Sample includes all available data from 2016.

So as to avoid artificially inflated metrics, all were obtained two hours after the end of the journal club. Participants were defined as anyone who posted an original tweet or retweeted during the session and were encouraged to tag their tweets with the hashtag \#JHMChat for post-discussion indexing and measurement. Because authors' or guests' popularity on Twitter may influence participation rates, we also assessed the number of followers for each participating author. Spearman's rank correlation was calculated (Microsoft ExcelTM) where appropriate.

\section{Altmetrics and Page Views}

As a means to measure exposure and dissemination external to Twitter, we assessed the change ("Delta") in the each article's Altmetric score, ${ }^{9}$ a digital-based metric that quantifies the attention received by a scientific publication on various online platforms including news, blogs, and social media. Delta Altmetric scores were calculated as the difference between the scores on the day of the session and two weeks after the respective session, with higher scores indicating greater global online discussion. By measuring the Altmetric score on the day of the discussion, we established a baseline score for comparison purposes. Additionally, this allowed us to better attribute any changes that may have occurred to the discussion itself.

Additionally, using information provided by the journal publisher (John Wiley \& Sons Publishing) in 2016, we assessed the effect of \#JHMChat on the number of article page views on the JHM website relative to the release of the electronic Table of Contents (eTOC). The eTOC release was chosen as it is historically associated with a high number of page views. In order to isolate the effect of \#JHMChat, we only reviewed months in which \#JHMChat was not held within three days of the $\mathrm{eTOC}$ release. Because JHM changed publishers in Janu- ary 2017, we only assessed page view data on 2016 sessions, as the new publisher lacked enhanced search optimization to obtain these data.

\section{Thematic Analysis}

In addition to the above measurements, a thematic analysis of each article was conducted to assess any common themes that would influence our chosen metrics. Themes were assessed and ascribed by one author (C.M.W.) and verified by another (V.M.A.).

\section{Participant and Author Experience}

To assess the participant experience, responses to a post-session CME questionnaire that assessed (1) overall quality, (2) comprehensiveness of the discussion, (3) whether the participant would recommend the chat to a colleague, and (4) whether participation would lead to practice-changing measures were reviewed. Registration of each session for CME was also quantified. Finally, each participating author was asked to fill out an electronic post-chat survey (SurveyMonkey ${ }^{\circledR}$ ) meant to assess the authors' experience with the journal club (Appendix).

\section{RESULTS}

Between October 2015 and November 2017, a total of 15 sessions were held with a mean of $2.17( \pm 0.583)$ million impressions/session, $499( \pm 129)$ total tweets/session, and $73( \pm 24)$ participants/session (compared to a range of 21-58 participants/session from other online journal clubs, where reported) with $7.2( \pm 2.0)$ tweets/participant (Table 1). The total number of participants for all sessions was 1,096. Participating authors had on average $1,389( \pm 2,714)$ followers, ranging from a low of 37 
I felt that the Twitter venue allowed me to discuss my manuscript with enough depth for participants to capture a good picture of what we did (eg, implementation processes and challenges, ways to overcome challenges, and creating culture change).

The JHMChat provided me with an opportunity to share my work with a different group of participants than with whom I routinely interact with.
The questions that were brought up by the moderator or listeners during the JHMChat helped me consider how I discuss my manuscript in the future.
I believe that the discussion during the JHMChat was informative to other participants.
I believe that by participating in the JHMChat, my manuscript achieved greater dissemination and visibility.
Overall, the JHMChat was a valuable experience for me
about new practice.

to a high of 10,376 (Appendix). No correlation between author following and number of participants $(r=0.19)$, impressions $(r=0.05)$, or change in Altmetric score $(r=0.17)$ was seen.

Thematic analysis revealed three predominant themes among the chosen articles: Value-based care (VBC), Quality and Patient Safety (QPS), and Medical Education (ME). Articles focused on VBC had the greatest number of impressions (mean \pm SD: 2.61 \pm 0.55 million) and participants (mean \pm SD: $90 \pm 12$ ), while OPS articles had the fewest impressions (mean \pm SD: $1.71 \pm 0.59$ million) and number of participants (mean \pm SD: $47 \pm 16$ ). The mean increase in the Altmetric score among all discussed articles was $14( \pm 12)$, from an average baseline of $30( \pm 37)$. Medical Education-themed articles appeared to garner the greatest increase in Altmetric scores, averaging an increase of 32 points, compared with an average baseline score of $31( \pm 32)$. In contrast, VBC and QPS articles averaged an increase of 8.6 and 8.4 points, from average baselines of $55( \pm 53)$ and $17( \pm 13)$, respectively. A twomonth analysis of JHM articles not included in these discussions, in which Altmetric scores were measured in the same way as those from the discussion, revealed a baseline Altmetric score of $27( \pm 24)$ with an average increase of $8( \pm 6)$ two weeks following the chat.

Four articles met the inclusion criteria for page view analysis and suggested that article page views increased to similar levels as the ETOC release (mean: 2,668 vs. 2,998, respectively; $P=.35$ ) (Figure). These increases equate to a $33 \%$ and $50 \%$ increase in average daily page views $(2,002)$ for the chat and eTOC release, respectively.

On average, $10( \pm 8.0)$ individuals/session registered for $\mathrm{CME}$, with 119 claiming CME credit in total. Forty-six percent (55/119) of participants completed the post-discussion questionnaire, with $93 \%$ and $87 \%$ reporting the sessions as 'very good' or 'excellent' with regard to overall quality and comprehensiveness of the session, respectively. Ninety-seven percent stated that they would recommend \#JHMChat to a fellow colleague, and 95\% stated that participation in the chat would change their practice patterns through any of the following: changing their personal practice, teaching others about the new practice, revising a protocol or institutional policy or procedure, or educating patients about the new practice (Table 2).

Ninety-three percent (14/15) of the participating authors responded to the post-discussion survey. All strongly agreed (5/5 on a Likert scale) that the venue allowed for an in-depth discussion about processes and challenges in conducting the study and allowed for greater dissemination and visibility of their work (5/5). Additionally, authors agreed that the journal club was a valuable experience for themselves (4.88/5) and other practitioners (4.88/5). Most agreed that the journal club allowed them to share their work with a different group of participants than usual (4.75/5) and that the experience changed how they would discuss their manuscripts in the future (4.75/5.0; Table 2).

\section{DISCUSSION}

The Twitter-based journal club \#JHMChat appears to increase social media awareness and dissemination of journal articles and was considered a useful engagement platform by both authors and participants.

Articles with a focus on VBC and ME had the greatest impact on dissemination metrics, particularly, total impressions and Altmetric scores, respectively. Given the strong presence 
and interest in these topics within Twitter and social media, these findings are not surprising. ${ }^{10,11}$ For example, over the past several years, the VBC movement has taken shape and grown alongside the expansion of social media, thus giving a space for this community to grow and engage. Of note, the cosponsorship relationship with the ABIM Foundation (which works closely with the Choosing Wisely ${ }^{\circledR}$ campaign) and the Costs of Care Organization could have influenced the participation and dissemination rates of VBC articles. Medical education articles were also popular and appeared to have increased uptake after chats, based on their Altmetric scores. This may be explained by the fact that medical educators have long utilized social media as a means to connect and engage within their community. ${ }^{12-14}$ It is also possible that the use of Twitter by trainees (residents, students) may have driven some of the dissemination of ME articles, as this group may not be regular subscribers to JHM.

Online journal clubs offer distinct advantages over traditional in-person journal clubs. First, online journal clubs allow for increased connectivity among online communities, bringing together participants from different geographic areas with diverse training and clinical experiences. Subsequently, this allows for the rapid exchange of both personal and organizational approaches to the topic of discussion..$^{15-17}$ Second, online journal clubs allow for continual access to the discussion material. For example, while the metrics used in this study only assessed active, synchronous participation, anecdotal evidence and feedback to the authors suggests that many individuals passively engaged by following along or reviewed the chat feed post hoc at their convenience. This asynchronous access is a quality not found in more traditional journal club formats. Finally, because online journal clubs commonly operate with a flattened hierarchy ${ }_{1}{ }^{18}$ they can break down access barriers to both the researchers who performed the study and thought leaders who commonly participate. ${ }^{17}$

Several insightful lessons were gleaned in the production and management of this online journal club. On the implementation side, promotion, preparation, and continued organization of an online journal club requires a fair amount of work. In this case, the required time and resources were provided by two social media editors in addition to administrative assistance from the SHM. The high attrition rate of online journal clubs over the years attests to these difficulties. ${ }^{24}$ Additionally, finding incentives to attract and sustain participation can be difficult, as we noted that neither CME nor author popularity (based on their Twitter following) appeared to influence engagement metrics (number of participants, total tweets, and tweets/participant). We also found that partnering with other journal club communities, in particular \#NephJC, lead to greater participation rates and impressions. Thus, leveraging connections and topics that span clinical domains may be one way to improve and broaden engagement within these forums. Finally, feedback from participants revealed that the timing of the journal club and the inability to have in-depth discussions, a characteristic commonly associated with traditional journal clubs, were problematic.
This study has several limitations. First, the metrics used to assess social media engagement and dissemination can be easily skewed. For instance, the activity of one or two individuals with large followings can dramatically influence the number of impressions, giving a falsely elevated sense of broad dissemination. Conversely, there may have been some participants who did not use the \#JHMChat hashtag, thus leading to an underestimation in these metrics. Second, while we report total impressions as a measure of dissemination, this metric represents possible interactions and does not guarantee interaction or visualization of that tweet. Additionally, we were unable to characterize our participants and their participation rates over time, as this information is not made available through Symplur ${ }^{\odot}$ analytics. Third, our page view assessment was limited to 2016 sessions only; therefore, these data may not be an accurate reflection of the impact of \#JHMChat on this metric. Fourth, given the marginal response rate to our CME questionnaire, a selection bias could have occurred. Finally, whether social media discussions such as online journal clubs act as leading indicators for future citations remains unclear, as some research has shown an association between increased Altmetric scores and increased citation rates, ${ }^{19-21}$ while others have not. ${ }^{22,23}$ Our study was not equipped to assess this correlation.

\section{CONCLUSION}

Online journal clubs create new opportunities to connect, engage, and disseminate medical research. These developing forums provide journal editors, researchers, patients, and clinicians with a means to connect and discuss research in ways that were not previously possible. In order to continue to evolve and grow, future research in online journal clubs should explore the downstream effects on citation rates, clinical uptake, and participant knowledge after the sessions.

\section{Acknowledgments}

The authors would like to thank Felicia Steele for her assistance in organizing and promoting the chats. Additionally, the authors would like to thank all the authors, guests, and participants who took time from their families, work, and daily lives to participate in these activities. Your time and presence were truly appreciated.

Disclosures: The authors of this article operate as the Social Media Editors (C.M.W., V.M.A.) and the Editor-in-Chief (A.A.) for the Journal of Hospital Medicine. Dr. Wray had full access to all the data in the project, takes responsibility for the integrity of the data, and the accuracy of the data analysis.

\section{References:}

1. Topf JM, Sparks MA, Phelan PJ, et al. The evolution of the journal club: from osler to twitter. Am J Kidney Dis Off J Natl Kidney Found. 2017;69(6):827-836. doi:10.1053/j.ajkd.2016.12.012

2. Thangasamy IA, Leveridge M, Davies BJ, Finelli A, Stork B, Woo HH. International urology journal club via Twitter: 12-month experience. Eur Urol. 2014;66(1):112-117. doi:10.1016/j.eururo.2014.01.034

3. Gardhouse Al, Budd L, Yang SYC, Wong CL. \#GeriMedJC: the Twitter complement to the traditional-format geriatric medicine journal club. J Am Geriatr Soc. 2017;65(6):1347-1351. doi:10.1111/jgs. 14920.

4. Duque L. How academics and researchers can get more out of social media. Harvard Business Review. https://hbr.org/2016/06/how-academics-and-re- 
searchers-can-get-more-out-of-social-media. Accessed November 9, 2017.

5. Wray CM, Arora VM. \#VisualAbstract: a revolution in communicating science? Ann Surg. 2017;266(6):e49-e50. doi:10.1097/SLA.0000000000002339.

6. Ibrahim AM. Seeing is believing: using visual abstracts to disseminate scientific research. Am J Gastroenterol. 2017:ajg2017268. doi:10.1038/ ajg.2017.268

7. \#JHMChat. http://shm.hospitalmedicine.org/acton/media/25526/jhmchat. Accessed November 9, 2017

8. \#JHMChat-healthcare social media. Symplur. https://www.symplur.com/ search/\%23JHMChat. Accessed November 9, 2017

9. Altmetric. Altmetric. https://www.altmetric.com/. Accessed November 9, 2017

10. value-based healthcare | Symplur. https://www.symplur.com/topic/value-based-healthcare/. Accessed November 17, 2017.

11. medical education | Symplur. https://www.symplur.com/topic/medical-education/. Accessed November 17, 2017.

12. Sterling $M$, Leung $P$, Wright $D$, Bishop TF. The use of social media in graduate medical education: a systematic review. Acad Med. 2017;92(7):1043. DOI:10.1097/ACM.0000000000001617.

13. Davis WM, Ho K, Last J. Advancing social media in medical education. CMA Can Med Assoc J. 2015;187(8):549-550. DOI:10.1503/cmaj.141417.

14. Hillman T, Sherbino J. Social media in medical education: a new pedagogica paradigm? Postgrad Med J. 2015;91(1080):544-545. doi:10.1136/postgradmedj-2015-133686.

15. Gerds AT, Chan T. Social media in hematology in 2017: dystopia, utopia, or somewhere in-between? Curr Hematol Malig Rep. 2017;12(6):582-591. doi:10.1007/s11899-017-0424-8.
16. Mehta N, Flickinger T. The times they are a-changin': academia, social media and the JGIM Twitter Journal Club. J Gen Intern Med. 2014;29(10):1317-1318. doi:10.1007/s11606-014-2976-9.

17. Chan T, Trueger NS, Roland D, Thoma B. Evidence-based medicine in the era of social media: scholarly engagement through participation and online interaction. CJEM. 2017:1-6. doi:10.1017/cem.2016.407.

18. Utengen A. The flattening of healthcare: breaking down of barriers in healthcare social media-twitter visualized. https://www.symplur.com/shorts/ the-flattening-of-healthcare-twitter-visualized/. Accessed November 8, 2017.

19. Thelwall M, Haustein S, Larivière V, Sugimoto CR. Do altmetrics work? Twitter and ten other social web services. PloS One. 2013;8(5):e64841. doi:10.1371/ journal.pone.0064841

20. Peoples BK, Midway SR, Sackett D, Lynch A, Cooney PB. Twitter predicts citation rates of ecological research. PloS One. 2016;11(11):e0166570. doi:10.1371/journal.pone.0166570.

21. Eysenbach G. Can tweets predict citations? Metrics of social impact based on Twitter and correlation with traditional metrics of scientific impact. J Med Internet Res. 2011;13(4):e123. doi:10.2196/jmir.2012.

22. Winter JCF de. The relationship between tweets, citations, and article views for PLOS ONE articles. Scientometrics. 2015;102(2):1773-1779. doi:10.1007/ s11192-014-1445-x.

23. Haustein $S$, Peters I, Sugimoto $C R$, Thelwall $M$, Larivière $V$. Tweeting biomedicine: an analysis of tweets and citations in the biomedical literature. J Assoc Inf Sci Technol. 2014;65(4):656-669. doi:10.1002/asi.23101.

24. Journal club. In: Wikipedia. 2017. https://en.wikipedia.org/w/index.php?title=Journal_club\&oldid=807037773. Accessed November 9, 2017. 